\title{
Evaluación de los programas de prevención en drogas en el marco de la política nacional de la juventud
}

Eladia Carolina Álvares ${ }^{1}$

Héctor Escalante ${ }^{2}$

\section{RESUMEN}

El consumo de drogas constituye un fenómeno emergente en Salud Pública, su impacto se ve en la calidad de vida de los jóvenes y deteriora la economía del Estado. La promulgación de una nueva ley y la creación del Instituto Nacional de la Juventud abren brecha para el trabajo de incidencia política, impulsando iniciativas de políticas públicas en pro de la juventud, como titulares de derecho en la legislación hondureña. Materiales y métodos: se analizaron desde el enfoque de derecho a la salud y la equidad 15 programas sobre prevención del consumo de drogas lícitas e ilícitas en los jóvenes de 12 a 30 años; y su vinculación con la Política Nacional de Juventud. Se realizó un análisis cuantitativo basado en un estudio documental, desde el inicio del Consejo Nacional de Juventud, la Ley Marco para el Desarrollo Integral, hasta la elaboración y promulgación de la política.

El Sistema Nacional de Juventud responsable de la gestión e Intervenciones estratégicas para la equidad social de los jóvenes en la prevención del consumo de drogas, no ha consolidado una estrategia que permita la disminución en el uso y abuso de drogas. La suscripción de acuerdos e implementación de programas se vinculan con los lineamientos estratégicos de la Política Nacional de Juventud, no obstante, no han alcanzado resultados esperados en el disfrute pleno de los derechos de la juventud. Con respecto a la ubicación de los programas, están en su mayoría en el área rural, enfocando sus acciones en procesos integrales de atención psicológica, espiritual, educativa y emprendedurismo. Asimismo, desempeñan funciones de protección social.

\footnotetext{
${ }^{1}$ Profesora , Escuela de Enfermería, Facultad de Ciencias Médicas, UNAH: c.alvarez.unah@gmail.com

2 Profesor investigador, Posgrado en Salud Pública, Facultad de Ciencias Médicas, UNAH: escalante.h@gmail.com
} 
Palabras clave: políticas, drogadicción, programas, jóvenes.

\section{ABSTRACT}

The use of drugs constitutes an emerging phenomenon, that affects the young boys and girls' quality of life and it also deteriorates the state economy. The enactment of a new law and the creation of the National Youth Institute opens a gap pen gap for advocacy work, promoting public policy initiatives towards youth as rights holders in Honduran legislation.

Materials and methods were analyzed from the perspective of the right to health and equity of 15 programs on the prevention of licit and illicit consumption of drugs in young people (12 to 30 years) and its relationship with the National Youth Policy. The study was based on a quantitative analysis, starting at the beginning of the National Youth Council, the Law for Integral Development framework, until the development and enactment of the policy.

The National System of Youth responsible for strategic management and social equity for young people in the prevention of drug interventions, has not established a strategy for the reduction in the use and abuse of drugs. The signing of agreements and implementation of programs is linked with the strategic guidelines of the National Youth Policy, however, they have not achieved expected results in the full enjoyment of the rights of youth. With respect to the location of programs they are mostly in rural areas, focusing its actions, spiritual, educational and entrepreneurialism integral processes of psychological care. They also play social protection functions.

Keywords: policies, drug addiction, programs, youth, prevention. 


\section{INTRODUCCIÓN}

Honduras es un país de población joven, constituyéndose este grupo poblacional en uno de los más importantes para la consolidación del desarrollo sostenible. La adolescencia y juventud son periodos frágiles y decisivos del ser humano, que plantean diversas preocupaciones y la creación de programas de prevención que sirvan de alternativas en la construcción de mejores oportunidades de vida, que potencie el pleno disfrute de la juventud en donde se presentan desigualdades sociales y económicas que han incrementado los niveles de pobreza. (Acuña, 2005)

Diversos estudios epidemiológicos han permitido apreciar que se trata de un problema cuya magnitud va en aumento, por lo que se vienen efectuando múltiples esfuerzos estatales para establecer medidas de control. De acuerdo con el Departamento de Investigación del Instituto Hondureño Alcoholismo Drogadicción y Farmacodependencia (IHADFA), en el cotejamiento de información recopilada, la mayoría de los trabajos de investigación han sido realizados en el medio urbano y en la población estudiantil; esto ha permitido establecer proyecciones sobre el consumo de drogas en la juventud, por lo que se reconoce la necesidad de realizar un estudio que se oriente en el análisis de la Política Nacional de Juventud y la incorporación de las mismas en los programas de prevención en drogas y que contribuyan a solventar un problema importante de salud pública.

Con este propósito, se ha elaborado un mapeo de los programas de prevención ubicados en los municipios del Distrito Central, Talanga y San Antonio de Oriente. Esta investigación se hizo mediante un análisis de las políticas públicas de juventud y los programas de prevención en drogas, en el cual se dan aportes científicos de la realidad del fenómeno de la drogadicción y el énfasis que se le ha dado para incluirlo en el marco de políticas públicas con la ejecución de los programas de prevención desde la perspectiva del derecho a la salud en la población de 12 a 30 años de edad.

\section{METODOLOGÍA}

El objetivo general de la presente investigación fue un análisis crítico de los programas de prevención del consumo de drogas licitas e ilícitas en las poblaciones de adolescentes y jóvenes y su vinculación con la Política Nacional de Juventud en tres municipios de Francisco Morazán, en septiembre y octubre del 2011. El tipo de 
estudio es exploratorio, descriptivo. Es exploratorio porque se realiza una revisión documental sobre la Política Nacional de Juventud (Juventud, 2007) siguiendo una secuencia cronológica desde la conformación del Consejo Nacional de Juventud, la Ley Marco para el Desarrollo Integral de la Juventud, hasta la elaboración y ejecución de la Política Nacional de Juventud. El propósito de esta revisión documental fue conocer si estos documentos contemplaban en sus ejes estratégicos el tema de prevención, promoción y protección de la drogadicción.

Para la recolección de datos se elaboró una matriz con la dirección, números teléfonos de los programas de prevención en drogas, también se hizo un mapa de georreferencia de las instituciones objeto de estudio.

Se visitaron 15 programas de prevención y rehabilitación en drogas identificados en los municipios de Talanga, San Antonio de Oriente y el Distrito Central del departamento de Francisco Morazán. La unidad de observación se compone por cada una de las instituciones de prevención de drogas y la unidad de análisis fueron los planes y políticas institucionales.

La recolecta de los datos se realizó en un periodo de 2 meses (septiembre y octubre de 2011), que comprendió la elaboración, aplicación de los instrumentos, el procesamiento de datos utilizando el programa estadístico SPSS, versión 18; en las que se registraron los datos anotados en los instrumentos con un análisis de fiabilidad según Alfa de Cron Bach de 0.829, que se aplicó al $100 \%$ de las encuestas aplicadas con intervalo de confianza del $95 \%$.

El análisis descriptivo se realzó por medio de distribución de frecuencias en Microsoft Excel XP 97- 2003, en donde se elaboraron cuadros y gráficos para variables cuantitativas. Posteriormente, se realizó una discusión del análisis de los resultados obtenidos.

\section{DISCUSIÓN}

Desde principios de los años setenta, Honduras ha venido adoptando diferentes estrategias para garantizar el acceso a los servicios de salud a la población, tales como el Compromiso de Adelaida Australia (Organización Mundial de la Salud, 2010), que enfatiza el acceso de todos a una alimentación y nutrición saludable y a la reducción de los riesgos por ingesta de alcohol y consumo de tabaco. 
Los jóvenes han sido caracterizados por la legislación hondureña como titulares de derechos e impulsados como actores estratégicos del desarrollo; garantizándoles el respeto y promoción de los derechos y deberes inherentes para propiciar su participación plena en el desarrollo de la nación.

Algunos incisos del cuestionario aplicado en este estudio se derivan directa e indirectamente de la Política Nacional de Juventud. En términos generales, existen algunos programas de prevención que se vinculan con los lineamientos estratégicos de la Política Nacional de Juventud, en la que se evidencia una separación entre esta y la implementación de los mismos, ya que el desconocimiento que los responsables de programa señalan con respecto a la Política Nacional de Juventud es una limitante para la aplicabilidad en pro de la juventud.

Los niveles de atención a la juventud que figuran en la agenda pública es el resultado de la reivindicación y logros que los jóvenes han alcanzado mediante procesos de construcción ciudadana, apoyados por la cooperación internacional como un elemento central para el desarrollo social. (Congreso Nacional de Honduras, 2006).

La Política Pública de Juventud define los entornos sociales que determinan las condiciones de vida de los jóvenes, sus familias y sus comunidades; por lo tanto, resulta clave para el proceso organizador del Estado que promueve, protege y hace efectivos los derechos humanos y se resume en un nuevo pacto social entre Gobierno y sociedad civil, en definición de prioridades políticas basadas en los principios que rigen la Política Nacional de Juventud, tales como la intersectorialidad, transversalidad, equidad generacional, de género, etnia, condición socioeconómica, fortalecimiento de la acción local, participación ciudadana y rendición de cuentas.

En tal sentido, frente al desafío de la pobreza, consumo de drogas y analfabetismo, entre otras limitantes, se pone de manifiesto la necesidad social de incorporar nuevas estrategias democráticas que apoyan en general esta meta de riesgo en la población joven. Por tal motivo, la expansión de capacidades humanas a través de políticas centradas en la equidad, la inversión en salud, educación, seguridad y cultura, son también maneras de luchar contra la pobreza, en la medida que aumentan las oportunidades de los jóvenes para llevar una vida digna.

Con respecto a la ubicación de los programas que trabajan en la prevención, rehabilitación y reinserción de los jóvenes vinculados al uso y consumo de drogas, en términos generales están ubicados en el área rural, dotados de espacios físicos disponibles para la recreación, talleres, panaderías, huertos, piscicultura, escuelas, entre 
otros, su ubicación geográfica en la zona no supone peligro para la integridad física 0 psíquica de los jóvenes según consta en el reglamento del Proyecto Victoria. Lo que tiene relación con los programas que se ubican a nivel internacional, (Irene M. Parada, 1994), considerando que la mayoría de los centros de rehabilitación de drogas pertenecen a organizaciones de desarrollo social, se encuentran lejos de los entornos urbanos y comúnmente están ubicados en las zonas rurales, tales como montañas, entre otros.

El presente estudio abarca 15 programas identificados en los municipios de Talanga, San Antonio de Oriente y el Distrito Central del Departamento de Francisco Morazán. El abordaje de las consultas y el análisis de las respuestas obtenidas por los programas que trabajan en la rehabilitación, reinserción y prevención en drogas han permitido conocer detalladamente su función social, su conocimiento y vinculación de la Política Nacional de Juventud, teniendo en cuenta que el problema de drogas, basados en la intervención y prevención, carecen de una discusión y asimilación amplia por parte de la sociedad, ante la complejidad del fenómeno y el grado de importancia que el Estado le presta.

Según los lineamientos de la Política Nacional de la Juventud establece que los programas de prevención de uso y abuso de drogas deben de contemplar: educación con calidad, estilos de vida saludable, protección social, trabajo digno, justicia como base de una cultura de paz, arte, deporte recreación y participación ciudadana (Grafico 1), los cuales tienen estrecha cohesión con la misión del ente regulador del Estado en esta temática (IHADFA). Cabe mencionar que todas estas instituciones estudiadas son de carácter privado.

Una vez egresados los jóvenes se les brinda seguimientos mediante programas que tienen incluidos en sus políticas estratégicas para la rehabilitación, reinserción, prevención. Se evalúan los componente con que trabajan estos programas en relación a lo que establece la Política Nacional de la Juventud, (Cuadro 2) se pudo observar que para cumplir estos propósitos los proyectos utilizan una variedad de métodos, entre ellos, terapia de grupo, terapias específicas de comportamiento para prevenir la recaída, participación en un grupo de ayuda mutua y llamadas por teléfono, entre otros. Estos hallazgos son similares con estudios realizados en otros países (María da Gloria Miotto Wright, 2008). 
Grafico 1. Aplicación de los lineamientos de la política Nacional de la Juventud en los programas de prevención en drogas, F, M, 2011

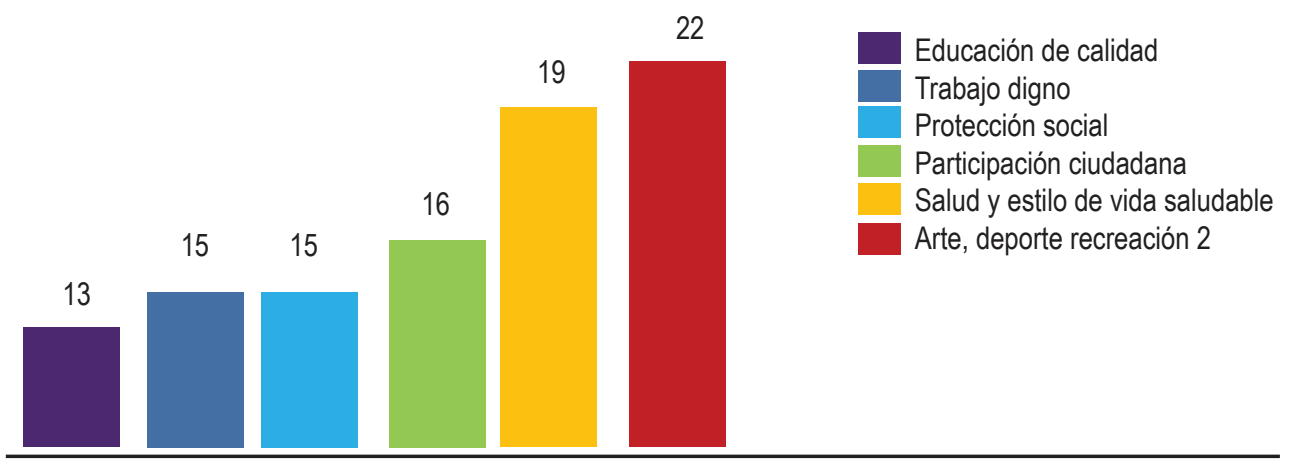

Grado de aplicación de los lineamientos estrategicos PNJ

Fuente: Elaboración propia

Grafico 2. Elementos de la misión de los programas de prevención en drogas contemplados en la Política Nacional de la Juventud

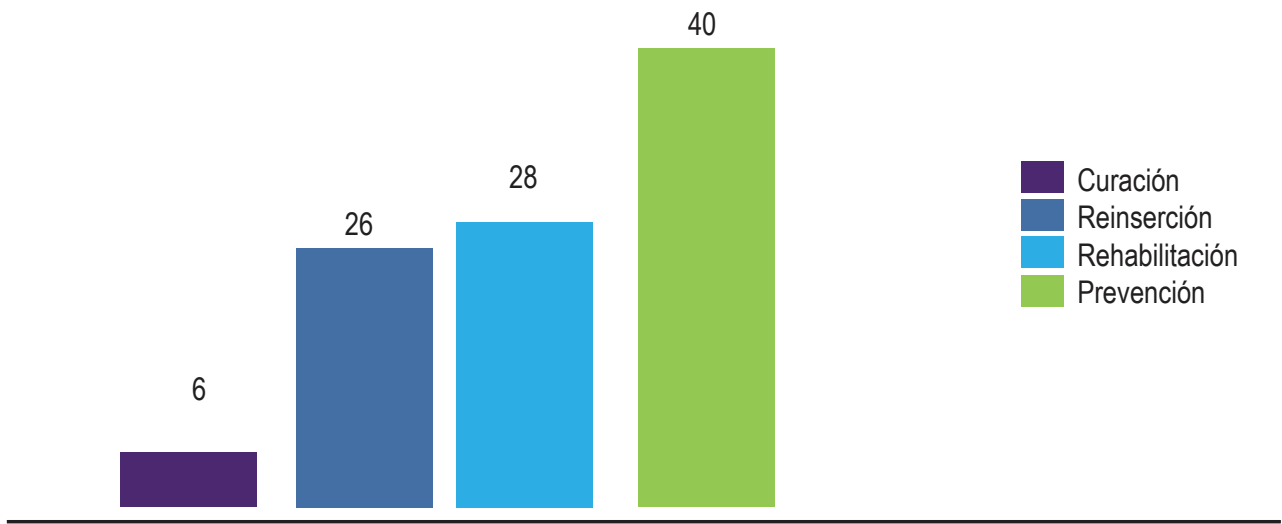

Elementos de la misión en los programas de prevención en drogas

Fuente: Elaboración propia 
Se considera importante que los programas de rehabilitación establezcan estrategias de redes sociales de asistencia trabajando con criterios multidisciplinarios e intersectoriales, en permanente comunicación con lugares de transición, apoyo comunitario y social; la rehabilitación viene después. En algunos programas de prevención a los jóvenes que ingresan como requisito necesitan realizarse exámenes médicos de laboratorio para descartar enfermedades infectocontagiosas y su ingreso se condiciona de acuerdo a la orden de solicitud y demanda.

El tratamiento y la rehabilitación de las personas con problemas de drogas, en términos generales, están bajo la responsabilidad de organizaciones no gubernamentales y servicios privados ubicados en áreas rurales y urbanas determinando estas variables la accesibilidad de los jóvenes y sus familiares para el ingreso y visitas. (Cuadro $3)$.

\section{Grafico 3. Ubicación geográfica de los programas de prevención de Droga. F.M, 2011}

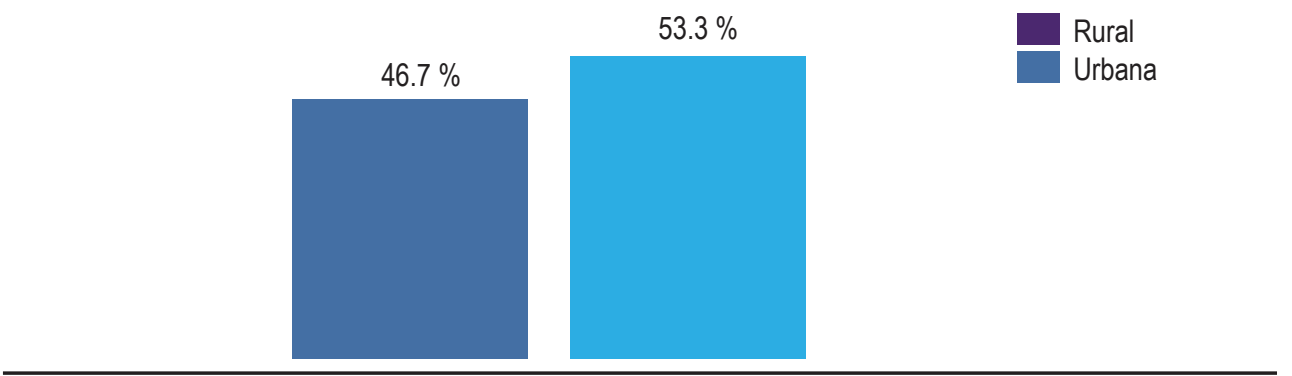

Ubicación geográfica

Fuente: Elaboración propia

Con relación al sistema educativo, diferentes programas de prevención tienen escuelas dentro de sus instalaciones, contribuyendo de esta manera a la formación profesional, disminuyendo con ello las inequidades sociales de este grupo poblacional considerado de alta vulnerabilidad social.

Acerca del presupuesto asignado en los fondos que perciben, en general, provienen principalmente de los fondos de las iglesias cristianas y católica, ya que son redes constituidas a nivel internacional en donde existen grupos voluntarios financiando los mismos. Asimismo, se estudiaron dos programas en donde el costo del proceso de rehabilitación lo realiza el familiar del interesado en rehabilitarse. 
Los jóvenes que sufren problemas relacionados con drogas suelen tener múltiples necesidades de tratamiento en una variedad de esferas personales, sociales y económicas, generadas por el abuso de drogas; estas se pueden tratar eficazmente si las personas afectadas tienen acceso a servicios de tratamiento y rehabilitación apropiados para atender sus necesidades y calidad, intensidad y duración suficiente. En la literatura revisada existe un estudio en México de 1990 a 1994 (Irene M. Parada, 1994), en donde se evidencia una mezcla pública y privada con relación al financiamiento. Tomando en cuenta que el presupuesto en salud es el mejor recurso para el desarrollo social, económico y personal que debe defender la conquista de condiciones cada vez más favorables para la salud y la vida.

El Estado ha dispuesto parte del presupuesto por medio del Instituto Hondureño para la Prevención del Alcoholismo, Drogadicción y Farmacodependencia (IHADFA), la Secretaría del Fondo de Población y el Despacho de la Primera Dama; cabe señalar que el mismo dispone de diferentes políticas y estrategias para tratar este problema; pero, en general, la mayoría de los programas objeto de estudio son privados, lo que excluye a los jóvenes para recibir atención.

Es importante señalar las limitantes que se tuvieron en la ejecución del proceso para realizar y finalizar el estudio, pues al hacer una revisión de la literatura se encontraron escasos estudios sobre este tema. Existen estudios que el CICAD12/ IHADFA ( 1988), ha realizado en algunos grupos con respecto a la prevalencia, incidencia, comportamientos y actitudes de los jóvenes hacia las drogas, especialmente en institutos públicos y privados, estudiantes de enfermería y medicina de la UNAH; lo que evidencia la necesidad de un abordaje más amplio de esta problemática. (IHAD$\mathrm{FA}, 2002$ )

Los profesionales señalaron el compromiso de los programas de velar para que ningún joven sea discriminado por motivo de sexo, raza, religión o discapacidad; este pensamiento del personal que labora tiene relación con el principio de equidad generacional, de género, etnia y condición socioeconómica que orienta la Política Pública de Juventud. 


\section{CONCLUSIONES}

1. La Política Nacional de Juventud establece su principio para dirigir sus acciones y garantizar el derecho a la salud y equidad de los jóvenes, focalizando lineamientos para la prevención de la violencia social y de género.

2. Respecto al uso y abuso de drogas, es prioridad planificar y ejecutar programas de prevención en este grupo poblacional que permita la protección y que garanticen el pleno goce de los derechos de los jóvenes de Honduras; teniendo en cuenta que no existen suficientes centros de prevención y que los existentes representan costos para sus beneficiarios, influyendo en los aspectos económicos y social para buscar una mejor atención en los problemas de drogadicción.

3. Se debe establecer una coordinación permanente y oficial en donde exista la articulación del Instituto Nacional de la Juventud en los programas de prevención en drogas, para realizar acciones conjuntas en la búsqueda de alternativas de solución en la disminución del uso y abuso de drogas.

4. Algunas organizaciones privadas que trabajan en la prevención en drogas, además de la prevención, desempeñan una función social orientando sus estrategias al rescate, protección social, educación, recreación, formación, vocacional y profesional para la rehabilitación e incorporación a la vida productiva de sus beneficiarios.

\section{RECOMENDACIONES}

1. El aumento acelerado del uso y abuso de las drogas y farmacodependientes por parte de los jóvenes amerita la ampliación de la cobertura y que esta asegure la universalidad y equidad del servicio, con inclusión, gratuita e integralidad en la atención.

2. El Estado debe promover la realización de estudios a través de investigaciones científicas, a fin de establecer medidas más acertadas en el tratamiento y eficientar los resultados terapéuticos a través de la priorización y gestión de acciones y asignación de recursos. 
3. Los órganos estatales que tienen funciones ligadas a la seguridad ciudadana, prevención primaria, educación, rehabilitación y reinserción social deben unir esfuerzos para consolidar ejes de atención como respuesta de prevención primaria, para los jóvenes involucrados en el uso y consumo de drogas y farmacodependientes.

\section{BIBLIOGRAFÍA}

Acuña, M.C. (2005). Exclusión, protección social y el derecho a la salud. Ginebra: OPS/OMS.

Alvarenga, E. (2002). Conocimientos y Actitudes y practicas sobre farmacodependencia en los estudiantes de las escuelas normales. Tegucigalpa, Honduras: IHADFA

Contraindropoulos, A. P. (2006). La ciencia entre las ciencias de la vida y las ciencias sociales. En elementos para una topografia del concepto de salud (págs. 30-32). Montreal Canada: Ruptures revista interdiciplinaria de la salud.

Naciones Unidas, Oficina contra la droga y el delito (2003). Manual sobre tratamiento del abuso de drogas, Viena.

Irene, M.; Parada, P.H. (1994). Financiamiento del programa de farmacodependencia. México: Salud Pública.

Instituto Nacional de Juventud (15 de noviembre de 2010). Política Nacional de Juventud. Tegucigalpa.

Poder Legislativo (16 de enero de 2006). Ley Marco para el Desarrollo Integral de la Juventud. La Gaceta, numero 30903.

Miotto, María da Gloria.; Wright, E. V. (2008). Las escuelas de enfermería, la investigación y el fenómeno de las drogas en América Latina. Colombia: Organización de los Estados Americanos.

Organización Panamericana de Salud. (2009). Monitoreo y análisis de los Procesos de Cambio y Reforma. Tegucigalpa.

Ugalde, E.G. (2009). La salud y la vida. Ecuador: OPS/OMS

Vittetoe, D. W. (2001). Estudio PACARDO. Tegucigalpa: IHADFA 